\title{
Indirect estimation of masses beyond collider reach in EFT
}

\section{Florian Goertz}

Max-Planck-Institut für Kernphysik,

Saupfercheckweg 1, 69117 Heidelberg, Germany

Theory Division, CERN,

1211 Geneva 23, Switzerland

E-mail: fgoertz@mpi-hd.mpg.de

ABSTRACT: We demonstrate how masses of new states, beyond direct experimental reach, could nevertheless be estimated in the framework of effective field theory (EFT), given broad assumptions on the underlying UV physics, however not sticking to a particular setup nor fixing the coupling strength of the scenario. The flat direction in the couplingvs.-mass plane ( $g_{*}$ vs. $M$ ) is lifted by studying correlations between observables that depend on operators with a different $\hbar$ scaling. We discuss the remaining model dependence (which is inherent even in the EFT approach to have control over the error due to the truncation of the power series), as well as prospects to test paradigms of UV physics. We provide an assessment of which correlations are best suited regarding sensitivity, give an overview of possible/expected effects in different observables, and demonstrate how perturbativity and direct search limits corner possible patterns of deviations from the SM in a given UV paradigm. In particular, given a certain pattern of deviations from the SM, we address the question whether we actually expect to see the new states at the LHC or the FCC, relying solely on EFT arguments.

Keywords: Beyond Standard Model, Effective Field Theories, Higgs Physics

ARXIV EPRINT: 1711.03162 


\section{Contents}

1 General introduction 1

2 Setup 2

3 Analysis + discussion $\quad 5$

4 Conclusions $\quad 14$

\section{General introduction}

Given the clear technical limitations in increasing the energy of collider experiments, it is important to try to access the properties of physics beyond the Standard Model (BSM) indirectly via precise measurements of observables at available energies. However, this approach features in general a significant limitation, since new degrees of freedom, entering as virtual particles in processes with characteristic energy scales below their production threshold, generically lead to corrections scaling like a ratio of their coupling and their mass. Acquiring information about their actual mass spectrum requires usually very specific assumptions about the coupling strength - which in reality however could span a huge range from very weak coupling $g_{*} \ll 1$ up to strong coupling, like $g_{*} \lesssim 4 \pi$. In this paper we point out how the different $\hbar$ scaling of various operators can be used to lift flat directions in the coupling vs. mass plane by examining more than one observable at a time, thus allowing to estimate systematically at which mass the new physics (NP) actually can be expected. Since the method will deliver an answer in principle up to a model dependent $\mathcal{O}(1)$ factor, complementing the power counting assumptions, we will mainly address the broad question whether we generically expect to see the new states directly at the LHC or the FCC.

The framework used for this analysis is the effective field theory (EFT) extension of the $\mathrm{SM}$, which is indeed the most general parametrization of NP that resides at energies much larger than both the electroweak scale $M_{\mathrm{EW}}$ and the characteristic scale $E$ probed by the experiment of interest. It can be fully formulated in terms of low mass (SM) fields, while the effect of the NP will manifest itself in the presence of operators with mass dimension $D>4$ in the effective Lagrangian $[1-3]$

$$
\mathcal{L}_{\text {eff }}=\mathcal{L}_{\mathrm{SM}}+\sum_{i} c_{i}^{(6)} \mathcal{O}_{i}^{(6)}+\sum_{j} c_{j}^{(8)} \mathcal{O}_{j}^{(8)}+\cdots
$$

where $\mathcal{L}_{\mathrm{SM}}$ is the SM Lagrangian, and we have assumed baryon and lepton number conservation. The operators $\mathcal{O}_{i}^{(D)}$ of canonical dimension $D$ consist of all (Poincaré invariant and hermitian) combinations of SM fields that respect local invariance under the (linearlyrealized) $\mathrm{SU}(3)_{c} \times \mathrm{SU}(2)_{L} \times \mathrm{U}(1)_{Y} \mathrm{SM}$ symmetry group. On dimensional grounds, operators 
of higher $D$ will be suppressed by larger powers of some fundamental mass scale $M \gg E$, associated to the new states that have been removed as propagating degrees of freedom in the low energy theory $\mathcal{L}_{\text {eff }}$ (i.e. integrated out in the path integral), with their effect being contained in the coefficients $c_{i}^{(6)}$ [4-19] (for reviews and further developments see, e.g., [20-28]). ${ }^{1}$ These coefficients allow to capture the effect of NP, no matter what are the exact details of the theory at higher energies. Determining them in experiment is a first step to understand the UV completion of the SM. On the other hand, as mentioned above, they generically depend only on ratios of couplings over masses, and not on masses alone, so naively it seems not possible to fix the spectrum of NP from such low energy observations, such as to know where to search for it. ${ }^{2}$

However, one needs to realize that not all operators depend on the very same ratio. In fact, from restoring $\hbar$ dimensions in the action and simple dimensional analysis, it is easy to convince oneself, that very generally an operator containing $n_{i}$ fields features a coefficient scaling as

$$
c_{i}^{(D)} \sim \frac{(\text { coupling })^{n_{i}-2}}{(\text { high mass scale })^{D-4}},
$$

given that the UV theory is perturbative (see, e.g., [30-32]). ${ }^{3}$ Analyzing the effect of more than one operator at a time can then provide information about $M$.

In fact, as we will study in detail below, exploring operators with a different field content allows us to gain sensitivity on different ratios of coupling over mass such as to solve for the latter. The broad assumptions required to entertain such correlations will be detailed in the following section. They correspond to a set of power counting rules, which are required in any case to assess the validity of the EFT setup, and do not include specific assumptions, such as on concrete coupling strengths. In this context, we will also scrutinize expected patterns of deviations in different UV paradigms, confronting them with collider observables in order to eventually rule out/favor certain model classes.

This article is organized as follows. In section 2, we will provide more details on the setup that forms the basis for our analysis, in section 3, we will perform the actual simultaneous study of different observables that will allow us to learn something on the underlying mass scale and to test UV paradigms, while we will conclude in section 4.

\section{Setup}

In the following, we will detail the power counting rules used in the analysis at hand. They basically correspond to the assumption of one new scale $M$ and one NP coupling $g_{*}$, in the spirit of the Strongly-Interacting Light Higgs (SILH) [30], and are fulfilled in a broad class of models where a weakly coupled narrow resonance (characterized by a single coupling

\footnotetext{
${ }^{1}$ This suppression allows for a truncation of the series at a certain $D$, assuring the predictivity of the setup.

${ }^{2}$ See also [29] for a recent discussion on the difference of new mass thresholds and (combined) interaction scales.

${ }^{3}$ Note that an additional suppressing factor (coupling $\left./ 4 \pi\right)^{2 L}$ can arise if the operator is only generated at the $L^{\text {th }}$-loop order.
} 
constant) is integrated out but as well in strongly coupled NP setups featuring a large $N$ description. We will comment on the presence of additional $\mathcal{O}(1)$ factors below. Note that the assumption of power counting rules is crucial in any EFT if one wants to assess its validity, since only after fixing the scaling of operators with couplings and masses can one start to estimate the effect of truncating the EFT series at a certain mass dimension (see, e.g., [33]). For example, in holographic composite Higgs models [34], the mass scale is set by the Kaluza-Klein (KK) mass $M \sim e^{-k r \pi} k \equiv M_{\mathrm{KK}}$ and the coupling-strength is given by the rescaled five-dimensional gauge coupling $g_{*} \sim g_{5} / \sqrt{2 \pi r}$, where $k$ is the $\mathrm{AdS}_{5}$ curvature and $r$ the compactification length of the fifth dimension.

In basic examples of integrating out a narrow resonance with a universal coupling to the $\mathrm{SM}$, the couplings entering the coefficients $c_{i}^{(D)}$ can in many cases indeed be identified with the single NP coupling $g_{*}$. On the other hand, in more complicated scenarios, interactions of the SM sector with NP might involve additional small (mixing) parameters and different operators might come with different effective couplings. In the classical SILH, in fact operators involving gauge bosons (or light fermions) feature in general smaller couplings $g_{V}<g_{*}$, due to the non-maximal mixing of the corresponding fields with the strong sector. ${ }^{4}$ Such suppressions can however be lifted in scenarios that complement the SILH extension of the SM. In a setup of vector-compositeness, dubbed Remedios [35], also gauge bosons couple in certain cases with the same strength to the strong sector as the Higgs, $g_{V}=g_{*}$. We will denote variants of well-known scenarios that feature just the latter difference with a bar, i.e., SILH, in the case discussed before. Beyond that, in a general description of a light Higgs, without identifying it with a Goldstone boson as in the SILH, but rather assuming the smallness of the electroweak scale is due to some other mechanism or an accident - the ALH - (loop) suppression factors of the SILH are not present (see [35]). The concrete scaling of operators in these two basic frameworks of BSM physics (including their 'Remedios' versions ${ }^{5}$ ) is summarized in table 1, together with the example of integrating out a scalar $\mathrm{SU}(2)_{L}$ doublet $S(1,2)_{1 / 2}$ with hypercharge $Y=1 / 2[36,37] .{ }^{6}$ The corresponding operators are defined in table 2, where we employ the SILH basis (with an adapted normalization) [30,35]. Note that we always assume minimal flavor violation (MFV) to be at work, dictating the flavor structure of the operators [38], such as the coefficients $\lambda^{4 f}$ or the yukawa couplings entering table 1. Four-fermion operators under investigation below will be assumed to feature left-handed quark currents for concreteness, resulting in $\lambda^{4 f} \rightarrow V_{t b} V_{t s}^{*}$ in the case of $b s$ transitions to leading approximation. ${ }^{7}$ Finally,

\footnotetext{
${ }^{4}$ Note also that, due to the assumption of minimal coupling as well as symmetry considerations, some operators in the SILH feature further (loop) suppression factors [30, 35].

${ }^{5} \mathrm{We}$ assume the Remedios+MCHM of [35], however the MCHM-like scaling entering in the last five columns of table 1 is not crucial for the following analysis.

${ }^{6}$ We thus consider $\lambda_{\varphi}=g_{*}^{2}$ and $y_{\varphi}^{f}=y_{f}$.

${ }^{7}$ This is for illustration and the generalization to other operators that are generated in the scenarios is straightforward. Note that MFV dictates the ratios of couplings accompanying different quark currents and we neglect subleading terms, suppressed by powers of $y_{i}^{2} / y_{t}^{2} \ll 1$. Moreover, it is assumed that chiralsymmetry breaking effects are mediated by SM-like Yukawa couplings, leading to the appearance of $y_{f}$ in $c_{y_{f}}$ (or that the same physics that generates the $D=4$ Yukawa couplings also generates $\mathcal{O}_{y_{f}}$, which is true in many BSM scenarios, such as in composite Higgs models). We will also comment on the concept of partial compositenss further below.
} 


\begin{tabular}{|c|c|c|c|c|c|c|c|c|c|}
\hline & $\mathcal{O}_{y_{f}}$ & $\mathcal{O}_{4 f}$ & $\mathcal{O}_{6}$ & $\mathcal{O}_{3 W, 3 G}$ & $\mathcal{O}_{B B, G G}$ & $\mathcal{O}_{W, B}$ & $\mathcal{O}_{2 W, 2 B, 2 G}$ & $\mathcal{O}_{H W, H B}$ & $\mathcal{O}_{H}$ \\
\hline$\overline{\mathrm{SILH}}$ & $y_{f} g_{*}^{2}$ & $\lambda^{4 f} g_{*}^{2}$ & $\frac{\bar{y}_{t}^{2}}{16 \pi^{2}} g_{*}^{4}$ & $\frac{g_{*}^{2}}{16 \pi^{2}} g_{*}$ & $\frac{y_{t}^{2}}{16 \pi^{2}} g_{V}^{2}$ & $g_{V}$ & 1 & $\frac{g_{*}^{2}}{16 \pi^{2}}\left(g, g^{\prime}\right)$ & $g_{*}^{2}$ \\
\hline $\mathrm{SILH}$ & $y_{f} g_{*}^{2}$ & $\lambda^{4 f} g_{*}^{2}$ & $\frac{y_{t}^{2}}{16} g_{*}^{4}$ & $\frac{g_{V}^{2}}{16 \pi^{2}} g_{V}$ & $\frac{y_{t}^{2}}{16 \pi^{2}} g_{V}^{2}$ & $g_{V}$ & $\frac{g_{V}^{2}}{g_{*}^{2}}$ & $\frac{g_{*}^{2}}{16 \pi^{2}} g_{V}$ & $g_{*}^{2}$ \\
\hline $\mathrm{ALH}$ & $y_{f} g_{*}^{2}$ & $\lambda^{4 f} g_{*}^{2}$ & $g_{*}^{4}$ & $g_{*}$ & $g_{V}^{2}$ & $g_{V}$ & 1 & $g, g^{\prime}$ & $g_{*}^{2}$ \\
\hline $\mathrm{ALH}$ & $y_{f} g_{*}^{2}$ & $\lambda^{4 f} g_{*}^{2}$ & $g_{*}^{4}$ & $\frac{g_{V}^{2}}{g_{*}^{2}} g_{V}$ & $g_{V}^{2}$ & $g_{V}$ & $\frac{g_{V}^{2}}{g_{*}^{2}}$ & $g_{V}$ & $g_{*}^{2}$ \\
\hline $\int[\phi]$ & $y_{f} g_{*}^{2}$ & $\lambda^{4 f} g_{*}^{2}$ & $g_{*}^{4}$ & $\frac{g^{2}}{16 \pi^{2}} \frac{g}{60}$ & \multicolumn{5}{|c}{$\lesssim(4 \pi)^{-2}$} \\
\hline
\end{tabular}

Table 1. Scaling of the coefficients of the various $D=6$ operators in terms of couplings, in the framework of the $\overline{\text { SILH }}$ (first line), the ordinary SILH (second line), the $\overline{\text { ALH }}$ (third line), the ALH (fourth line), and when integrating out a narrow scalar $S(1,2)_{1 / 2}$ (fifth line). $\lambda^{4 f}$ denotes the flavor structure, see text for details.

\begin{tabular}{|c|c|}
\hline $\mathcal{O}_{y_{f}}=|H|^{2} \bar{f}_{L} H f_{R}$ & $\mathcal{O}_{V V}=|H|^{2} V_{\mu \nu}^{a} V^{a \mu \nu}$ \\
\hline $\mathcal{O}_{4 f}=\bar{f} \gamma^{\mu} f \bar{f} \gamma_{\mu} f$ & $\mathcal{O}_{V}=\frac{i}{2}\left(H^{\dagger} \sigma^{a} \overleftrightarrow{D}^{\mu} H\right) D^{\nu} W_{\mu \nu}^{a}$ \\
\hline $\mathcal{O}_{6}=|H|^{6}$ & $\mathcal{O}_{2 V}=-\frac{1}{2}\left(D_{\rho} V_{\mu \nu}^{a}\right)^{2}$ \\
\hline $\mathcal{O}_{3 V}=\frac{1}{3 !} F_{a b c} V_{\mu}^{a \nu} V_{\nu \rho}^{b} V^{c \rho \mu}$ & $\mathcal{O}_{H V}=i\left(D^{\mu} H\right)^{\dagger} \sigma^{a}\left(D^{\nu} H\right) V_{\mu \nu}^{a}$ \\
\hline $\mathcal{O}_{H}=\frac{1}{2}\left(\partial^{\mu}|H|^{2}\right)^{2}$ \\
\hline
\end{tabular}

Table 2. The operators under consideration, where $3 V=3 W, 3 G ; \quad V V=B B, G G$; $V=B, W ; 2 V=2 B, 2 W, 2 G ; H V=H B, H W$. Moreover, $a, b, c=1, \ldots, 8 ; 1, \ldots, 3 ; \varnothing$, for $\mathrm{SU}(3), \mathrm{SU}(2)_{L}, \mathrm{U}(1)_{Y}$, respectively, with $F_{a b c}=f_{a b c}, \epsilon_{a b c}, 1$ the corresponding structure constants (and clearly the $\sigma^{a}$ matrices absent in the case of $V=B$ as well as $D=\partial$ when acting on $B_{\mu \nu}$ ). Note that $f$ denotes, schematically, fermion fields.

we will comment on the scenario where $g_{V} \rightarrow g_{\mathrm{SM}}$ in footnote 8 .

We note from table 1 that in general strong correlations (via $g_{*}$ ) exist between the operators $\mathcal{O}_{y_{f}}, \mathcal{O}_{4 f}, \mathcal{O}_{6}$ (and $\mathcal{O}_{3 V}$ in the case of the Remedios setup), which have the same form in many scenarios, as emphasized by the shades of gray. We will thus consider measurable quantities that are transparently related to these operators in the following. Two diverse scenarios (visualized by orange and blue dashed lines) can be identified, which can be mapped to two benchmarks for the analysis of this article, capturing basically all models at hand concerning the class of operators under consideration. They are distinguished by the assumption whether $\mathcal{O}_{6}$ is tree or loop generated and summarized in table 3, denoted by capital letters $\mathbf{A}$ and $\mathbf{B} .^{8}$

\footnotetext{
${ }^{8}$ The given scaling in the case of $\mathcal{O}_{3 V}$ holds only in the Remedios setups, where $g_{V}=g_{*}$. While other correlations are rather robust, those including this operator will depend on this assumption. Note moreover, that in the original version of the SILH proposal [30] vectors were assumed to be weakly coupled — featuring exactly the SM gauge couplings - which would lead to the replacement $g_{V} \rightarrow g, g^{\prime}, g_{s}$ in columns $4-8$ in the second line of table 1 , for $V=W, B, G$, respectively. Clearly, making such a specific assumption on the value of couplings entering the NP terms could let us hope to be able to determine $M$, in this particular scenario, via processes involving gauge bosons. The analysis presented here on the one hand considers the generalized context where all NP terms could appear with a NP coupling - $g_{V}$ in the case of gauge bosons - (focusing on operators with a 'universal' scaling), but beyond that particularly envisages effects in operators without gauge bosons, $\mathcal{O}_{y_{f}}, \mathcal{O}_{4 f}$, and $\mathcal{O}_{6}$, with still ample room for NP. Moreover, TGC measurements and Higgs decays are often only sensitive to combinations of operators with different scaling, like $c_{W, B}$ and $c_{2 W, 2 B}$.
} 


\begin{tabular}{|c|c|c|c|c|}
\hline & $\mathcal{O}_{y_{f}}$ & $\mathcal{O}_{4 f}$ & $\mathcal{O}_{6}$ & $\mathcal{O}_{3 W, 3 G}$ \\
\hline $\mathbf{A}$ & $y_{f} g_{*}^{2}$ & $\lambda^{4 f} g_{*}^{2}$ & $g_{*}^{4}$ & $g_{*}$ \\
\hline $\mathbf{B}$ & $y_{f} g_{*}^{2}$ & $\lambda^{4 f} g_{*}^{2}$ & $\frac{y_{t}^{2}}{16 \pi^{2}} g_{*}^{4}$ & $\frac{g_{*}^{2}}{16 \pi^{2}} g_{*}$ \\
\hline
\end{tabular}

Table 3. Operators that can be basically divided in two classes of scalings, i.e., A: ALH-like and B: SILH-like. See text for details.

In the following section, we will detail how these scenarios can be tested by studying correlations between observables. In particular, we will discuss which patters of deviations are expected and how they would allow us to estimate NP masses, beyond collider reach, and which patterns would disfavor given UV paradigms. In fact, as we will work out below, accessing two operators at a time will allow for a 'model-independent' direct estimation of the NP mass $M$, valid in a large class of NP frameworks - with the only remaining freedom being (besides the aforementioned $\mathcal{O}(1)$ factor) the question whether one is in Scenario A or in Scenario B .

The latter information can however be obtained by including a third observable, a procedure that we will explicitly go through at the end of this article. Indeed, considering simultaneously measurements of the coefficients of two operators will give us a handle both on $M$ and $g_{*}$, which leads to a distinct prediction/estimation for the remaining coefficients in both scenarios, which can be confronted with bounds, eventually allowing us to asses which of the scenarios is viable, and which is disfavored.

\section{Analysis + discussion}

We will now study in detail how measurable quantities, that depend in a simple way on the operators identified in section 2, can be employed to estimate the ballpark of the mass of new states, even in case the available energy does not suffice to produce them directly. Let us start by exploring how simultaneous measurements of more than one such quantity can be used to lift the flat direction in $g_{*}$ vs. $M$ and examine the resulting sensitivity on $M$.

Consider two operators $\mathcal{O}_{1}$ and $\mathcal{O}_{2}$, whose coefficients, $c_{1} \sim g_{*}^{y_{1}} / M^{2}$ and $c_{2} \sim g_{*}^{y_{2}} / M^{2}$, feature a different scaling in $g_{*}, y_{1} \neq y_{2}$. Now, assume these coefficients are extracted from measurements, resulting in $c_{1}=X_{1}$ and $c_{2}=X_{2}$, with $X_{1,2}$ featuring mass dimensions $D=-2$. We can now solve the simple system of equations (neglecting loop factors, which can be implemented trivially)

$$
\frac{g_{*}^{y_{1}}}{M^{2}} \sim X_{1}, \quad \frac{g_{*}^{y_{2}}}{M^{2}} \sim X_{2}
$$

for $M$, leading (for $y_{1} \neq y_{2}$ ) to

$$
M \sim\left(\frac{X_{1}^{y_{2}}}{X_{2}^{y_{1}}}\right)^{\frac{1}{2\left(y_{1}-y_{2}\right)}} .
$$

We observe that in general the best sensitivity regarding NP masses results from studying pairs of operators that feature powers of $g_{*}$ that are large for each of the operators, but close 
to each other, $\Delta_{y} \equiv\left|y_{1}-y_{2}\right| \sim 1$. In Scenario $\mathbf{B}$, this would correspond for example to the pair of operators $\mathcal{O}_{6}$ and $\mathcal{O}_{3 V}$ (which are however loop suppressed, limiting the effects due to perturbativity), that feature $\Delta_{y}=1$ and would lead to a sensitivity $M \approx\left(X_{1}^{3 / 2} / X_{2}^{2}\right)$. Moreover, the sensitivity increases if the operator that features the smaller power of $g_{*}$ becomes stronger constrained. On the other hand, confronting measurements of $c_{y_{f}}$ and $c_{4 f}$ leads to no sensitivity at all in both scenarios - concerning the operators in table 3 , at least a measurement of $c_{6}$ or $c_{3 V}$ needs to be involved.

A general procedure to estimate the NP mass $M$ (or, more conservatively, to asses the detectability at colliders - see below), given a set of measurements, is now as follows

1. Consider a pair of measurements that determines two coefficients in table 3 and solve for $M$ via relation (3.2), employing the results presented in figures $1-5$, both for Scenario $\mathbf{A}$ and $\mathbf{B}$.

2. Solve for $g_{*}$ via eqs. (3.1), derive predictions for the remaining pseudo-observables, and confront them with measurements. Drop the scenario that is (experimentally) excluded or inconsistent with EFT assumptions or perturbativity (too small $M$, too large $\left.g_{*}\right) \cdot{ }^{9}$

3. The remaining solution provides a direct ballpark-estimate for the mass of new particles, completing the SM.

While not involving $\lambda_{Z}$ at the beginning avoids assumptions regarding vector-boson couplings, it can help to discriminate between the different setups in the end. In fact, if at some point in the procedure above one encounters a significant contradiction, the corresponding underlying hypothesis (e.g., the SILH with assumptions detailed before) can be basically excluded to be realized in nature.

We now move to a numerical study of the sensitivities to NP masses $M$, considering (hypothetical) measurements of

1) a relative shift in yukawa couplings $\delta y_{f}$

2) the coefficient $C_{9}$ of the four-fermion operator ${ }^{10} \mathcal{O}_{9} \equiv \frac{4 G_{F}}{\sqrt{2}} V_{t b} V_{t s}^{*} \frac{\alpha}{4 \pi}\left(\bar{s}_{L} \gamma_{\mu} b_{L}\right)\left(\bar{\ell} \gamma^{\mu} \ell\right)$

3) a relative deviation in the Higgs self coupling $\delta \lambda$

4) the triple-gauge coupling (TGC) $\lambda_{Z}$.

\footnotetext{
${ }^{9}$ In the setup considered, there are basically two expansions - one in higher dimensional operators suppressed by a mass scale $M$ and the usual expansion in the coupling parameter (of the UV theory), $g^{*}$. If the latter exceeds $g^{*} \lesssim 4 \pi$, the UV theory becomes non-perturbative and the matching to the EFT can no longer be trusted. Loops contributing to a certain observable with more powers of $g^{*}$ would not be suppressed anymore. Thus, such parameter points should be discarded, see below.

${ }^{10}$ We take the operator $\mathcal{O}_{9}$ as an example since several current anomalies hint to a non-zero value of its coefficient, $C_{9} \sim-1$ (see, e.g., [39-42]). Although not all scenarios considered here can address the experimental observation in a fully consistent way, the case can serve as an illustrative example for the method in any case.
} 

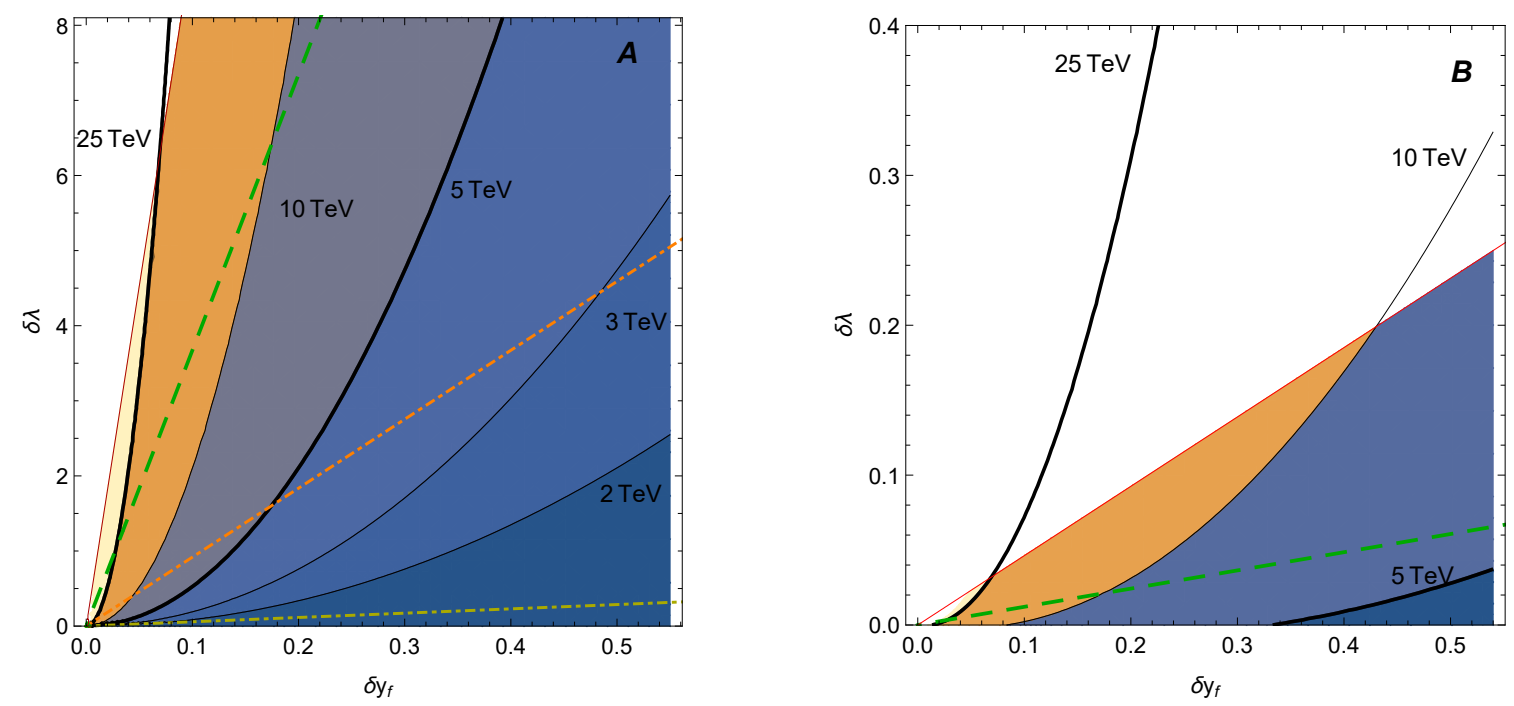

Figure 1. Estimated (maximal) NP mass $M_{\max }$ in dependence on the variation in the Yukawa couplings $\left(\delta y_{f}\right)$ and in the triple-Higgs self coupling $(\delta \lambda)$ in Scenario $\mathbf{A}$ (left) and $\mathbf{B}$ (right). The colored lines denote NP couplings of $g_{*}=1,4,8,4 \pi$, respectively (from yellow to red). See text for details.

These quantities are related to the coefficients of the operators in table 3 as (see, e.g., [27]) $)^{11}$

$$
\begin{aligned}
\delta y_{f} & =v^{3} /\left(\sqrt{2} m_{f}\right) c_{y_{f}} \\
C_{9} & =\frac{\sqrt{2} \pi}{\alpha G_{F} V_{t b} V_{t s}^{*}} c_{s_{L b_{L} \ell \ell}} \\
\delta \lambda & =2 v^{4} / m_{h}^{2} c_{6} \\
\lambda_{Z} & =-6 g^{2} m_{W}^{2} c_{3 W} .
\end{aligned}
$$

In that context, recall that $c_{s_{L} b_{L} \ell \ell}$ is assumed to scale like $V_{t b} V_{t s}^{*} g_{*}^{2}$, respecting MFV (such a structure is viable to allow for considerable effects in $C_{9}$, without being directly excluded from other measurements in flavor physics). A similar scaling holds in the case of partial compositeness, with the left-handed (b) quarks and leptons coupled significantly to the composite sector [43].

The expected sensitivities at the end of the high luminosity LHC (HL-LHC) run are $\delta y_{f} \sim 5 \%$, for $f=(t), b, \tau$ (see, e.g., [44]), $\delta \lambda \sim(20-30) \%$ [45-47], and $\lambda_{Z} \sim 10^{-3}[48-50],{ }^{12}$ which will set the ballpark for the hypothetical measurements considered below. These values could still be improved, for example by the ILC, which could allow for $\delta y_{f} \sim 1 \%$, for $f=b, c, \tau$ [44], $\delta \lambda \sim 10 \%$ [51], and $\lambda_{Z} \sim 10^{-4}$ [48] (see also [52, 53]). For $C_{9}$, on the

\footnotetext{
${ }^{11}$ Note that $\delta y_{f}$ and $\delta \lambda$ also receive contributions from a non-vanishing $c_{H}$. In the former case, these can be included simply by rescaling $\delta y_{f}$ by a factor of $3 / 2$ [27], which is accounted for in our numerical analysis (and for uniformity/simplicity we adjust similarly $y_{\varphi}^{f} \rightarrow 3 / 2 y_{f}$ for the scalar resonance). In the latter case, for Scenario $\mathbf{A}$ the effect is suppressed by the ratio of the (small) SM-like trilinear self coupling over the NP coupling squared, $\lambda / g_{*}^{2}$, and thus basically negligible for $g_{*} \gtrsim 1$. Since the interesting parameter space just features this range of couplings in all cases where $\delta \lambda$ is involved, this effect can be discarded. In Scenario $\mathbf{B}$, we include the impact of $c_{H}$ by adding a term $-\frac{3}{2} v^{2} c_{H}$ to the third line of eq. (3.3).

${ }^{12}$ Note that current experimental constraints are already at the level of $\lambda_{Z} \sim 3 \%$.
} 
other hand, we consider a value of $C_{9} \sim-1$, as suggested by experimental anomalies in $B$ physics, see footnote 10 .

In figures $1-5$, we finally explore the predictions for these quantities, induced by nonzero values of the corresponding coefficients, both for Scenario A and B. Employing relations (3.2) and (3.3), we can in principle draw iso-contours of constant $M$ (dividing regions of different color) in two-dimensional planes spanned by the different (pseudo-)observables, where we can determine the heavy mass $M$ via a combined measurement of the latter. However, taking into account that a numerical factor $N_{\mathrm{UV}}$ can enter the relations of table 3 when matching an explicit UV model following a certain power counting to the EFT, we rather draw isocontours of a maximal expected mass $M_{\max }$ that can generate a certain pattern, under a conservative assumption for $N_{\mathrm{UV}}$ augmenting the coupling over mass ratios and address the more basic question of whether this indicates the possibility to discover NP at the LHC (or FCC). To be conservative, we will either multiply an EFT coefficient entering the plots by $N_{\mathrm{UV}}$ or divide by the same factor, depending on which individual choice for the two coefficients maximizes the predicted mass.

In the following we consider $N_{\mathrm{UV}}=3$, being moderately large, while not yet spoiling the power-counting assumptions of the scenario (which would make such a counting useless in general). As it will turn out, even including such a factor, it will still be possible to find situations where a potential combined indirect measurement will basically guarantee a direct discovery at current/future colliders, or where a discovery becomes very unlikely in a given power counting. With a slight abuse of notation, we plot the absolute values of the corresponding quantities, keeping in mind that the signs of the various coefficients might vary.

We start by studying the correlations between $\delta y_{f}$ and $\delta \lambda$, visualized in figure 1 . We also present, as colored lines, the values of the coupling $g_{*}$ scanning the planes, where the dot-dashed yellow and orange lines correspond to NP couplings of $g_{*}=1,4$, respectively. The green dashed line visualizes the boundary of $g_{*}=8$, beyond which perturbation theory becomes problematic, while the red line, corresponding to $g_{*}=4 \pi$, signals the complete breakdown of perturbation theory. We thus do not draw the colored regions beyond this point. Finally, the thick black lines indicate the ballpark threshold for discovery of $5 \mathrm{TeV}$ $(25 \mathrm{TeV})$ at the LHC (FCC) [54].

Looking at the left panel of the figure, representing Scenario A, we find for example that the observation of a moderate deviation in Yukawa couplings of

- $\delta y_{f}=35 \%$ together with a change in the trilinear Higgs self coupling of $\delta \lambda=100 \%$ indicates that the NP resides not higher than $M_{\max } \sim 2 \mathrm{TeV}$ and thus is strongly expected to be discovered at LHC (in particular since $g_{*}>1$ )..$^{13}$

Observing on the other hand the same

- $\delta y_{f}=35 \%$ together with a larger $\delta \lambda \gtrsim 7$ leads to $M_{\max } \sim 6 \mathrm{TeV}$, leaving direct LHC reach.

\footnotetext{
${ }^{13}$ We have checked that the latter statement is rather robust and even still holds basically if we double the "OOO(1)" factor to $N_{\mathrm{UV}}=6$. However, we stress again that while it is in fact a strong expectation, assuming that the power counting receives no 'unnaturally' large corrections, it is no strict 'prove'.
} 
Turned around, in case of no direct discovery, seeing an effect in $\delta y_{f}$ requires a sizable correction $\delta \lambda$, as is evident from the plot due to the, perhaps naively counter-intuitive (yet power-counting understandable), increase of $M_{\max }$ with $\delta \lambda$, which is due to the peculiar scaling of the latter with a large power of $g_{*}$. For example, if a large $\delta y_{f} \approx 50 \%$ would be observed, while the self coupling would be constrained to $\delta \lambda<5$ (and no new physics would appear below $3 \mathrm{TeV}$ ), the large class of NP described by Scenario A could be basically excluded.

Regarding the FCC potential, its large mass reach would allow for a 'predicted' (expected) discovery for a much larger region in $\delta y_{f}$ vs. $\delta \lambda$ effects. Here, even a $\delta y_{f}=3 \%$, together with, say, $\delta \lambda \sim 100 \%$ would lead to NP within reach, while we could not say anything conclusive about direct LHC discoveries since this point would only come into expected reach for the latter collider if we could constrain $\delta \lambda$ below $5 \%$, which is unrealistic at the LHC (and planned colliders).

While we observe that in principle sizable effects in the Higgs self coupling are possible without low NP masses and evading effects in other observables - due to the scaling with the fourth power of a potentially large coupling $g_{*}$ — the large $\delta \lambda$ direction is still limited by perturbativity since a too large self coupling for constant $\delta y_{f}$ will eventually enforce non-perturbative $g_{*}$. This holds in particular for any observation of a sizable $\delta \lambda$ together with no observable effect in $\delta y_{f}$, at the left of the dashed green line, which is not possible in perturbation theory in the Scenario under consideration. ${ }^{14}$

Note that this becomes more extreme in Scenario $\mathbf{B}$, where $\delta \lambda$ is loop generated. For example (assuming $N_{\mathrm{UV}}=3$ ) even a $40 \%$ deviation in Yukawa couplings would allow at most for a $5 \%$ effect in the trilinear coupling, as can be seen from the right plot in figure 1, where deviations are thus doomed to be basically unobservable given already present constraints on other couplings (with the conclusion being similar for $N_{\mathrm{UV}}=1$ ). A different experimental result would exclude the SILH.

Regarding NP masses, here we find that a LHC discovery is hard to predict indirectly, unless borderline large effects in Yukawa couplings of $\delta y_{f} \gtrsim 50 \%$ would be about to be discovered, while changes in the Higgs self coupling would be found at the $(5-10) \%$ level (at most), which would indicate relatively low $M_{\max } \sim 5 \mathrm{TeV}$.

If we wanted to be more ambitious, we could turn a combined measurement of pseudoobservables into a real numerical estimate of the NP mass (setting $N_{\mathrm{UV}} \rightarrow 1$ ) - beyond more robust estimations of ranges lying completely inside or outside collider reaches, presented before. Given the caveats discussed, we present them here only to get a feeling for the ballpark of the expected NP mass, that can reside far beyond direct collider reach, yet being estimatable with the presented method. The maximally reachable sensitivity (respecting $g_{*}<8$ and experimental prospects) appears for the case at hand in Scenario A for $\delta y_{f}=1 \%, \delta \lambda \sim 3$ and leads to $M \sim 25 \mathrm{TeV}$. The corresponding values for all pairs of pseudo-observables for both scenarios will be summarized in table 4 .

We now move to figure 2, where we display the correlation between effects in the fourfermion operator $\mathcal{O}_{9}$ and $\delta \lambda$. Beginning again with Scenario $\mathbf{A}$, shown in the upper left

\footnotetext{
${ }^{14}$ For a different, general discussion on the potential size of $\delta \lambda$, see [55].
} 

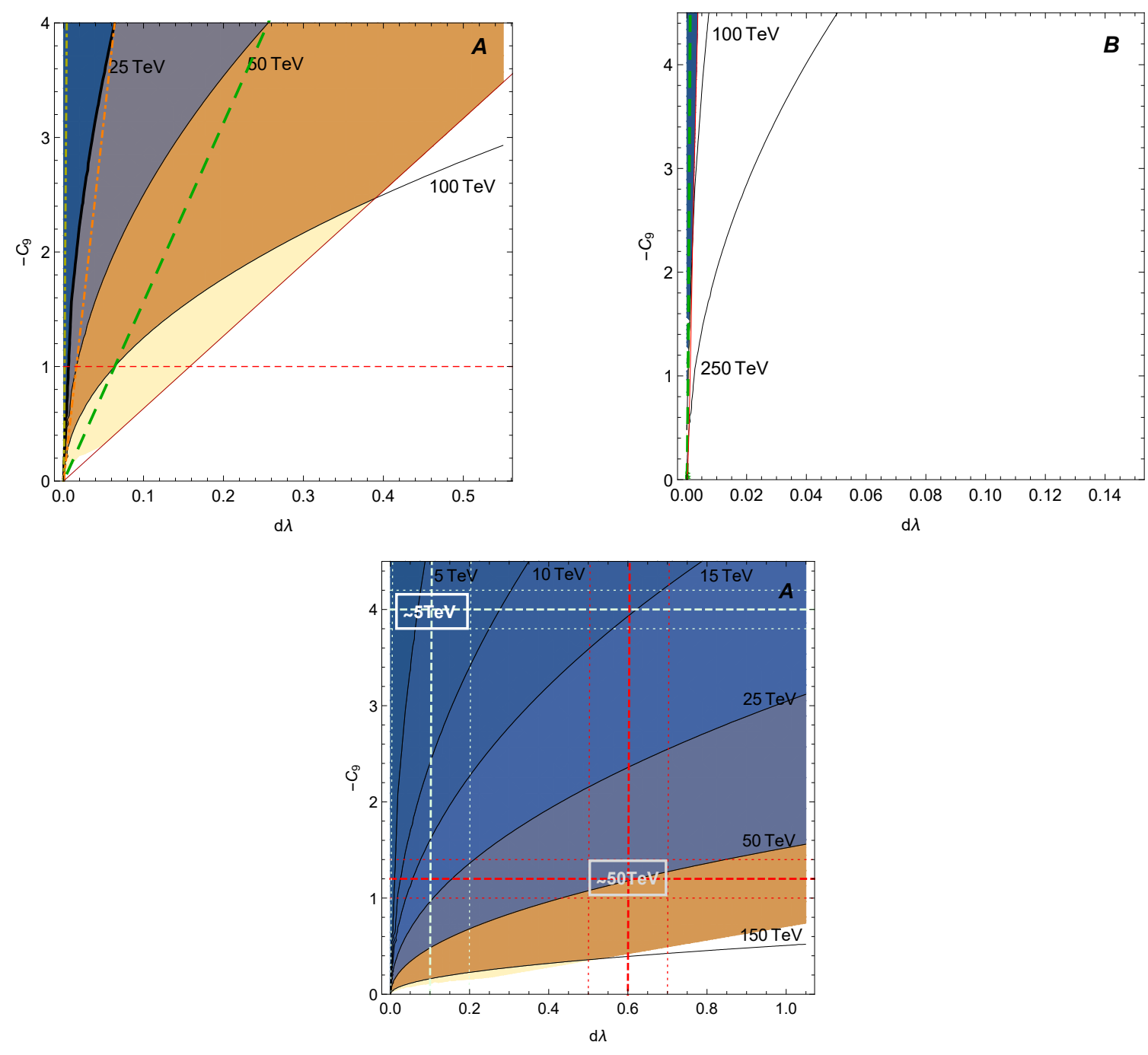

Figure 2. Estimated (maximal) NP mass $M_{\max }$ in dependence on the variation in the triple-Higgs self coupling $(\delta \lambda)$ and the coefficient of the four fermion operator $\mathcal{O}_{9}\left(C_{9}\right)$ in Scenario $\mathbf{A}$ (upper panel, left) and B (upper panel, right). The colored lines denote NP couplings of $g_{*}=1,4,8,4 \pi$, respectively (from yellow to red). The lower panel includes two hypothetical measurements of a signal in the $\lambda_{Z}-c_{9}$ plane, setting $N_{\mathrm{UV}} \rightarrow 1$, and illustrates the correspondingly extracted masses of new particles in Scenario A. See text for details.

panel, we observe that - barring unreasonably large $\left|C_{9}\right|$ - a robustly expected discovery is not possible to predict since all testable values for $\delta \lambda$ can easily feature NP masses beyond $25 \mathrm{TeV}$.

Even more, for Scenario $\mathbf{B}$ any halfway reasonable value for $C_{9}$ leads to a completely untestable $\delta \lambda$ and thus no information — beyond that statement — is available from this pair.

We will thus quickly turn to the next plane of observables, however before still comment again on the extraction of ballpark values for the mass of a new state, considering the plot 

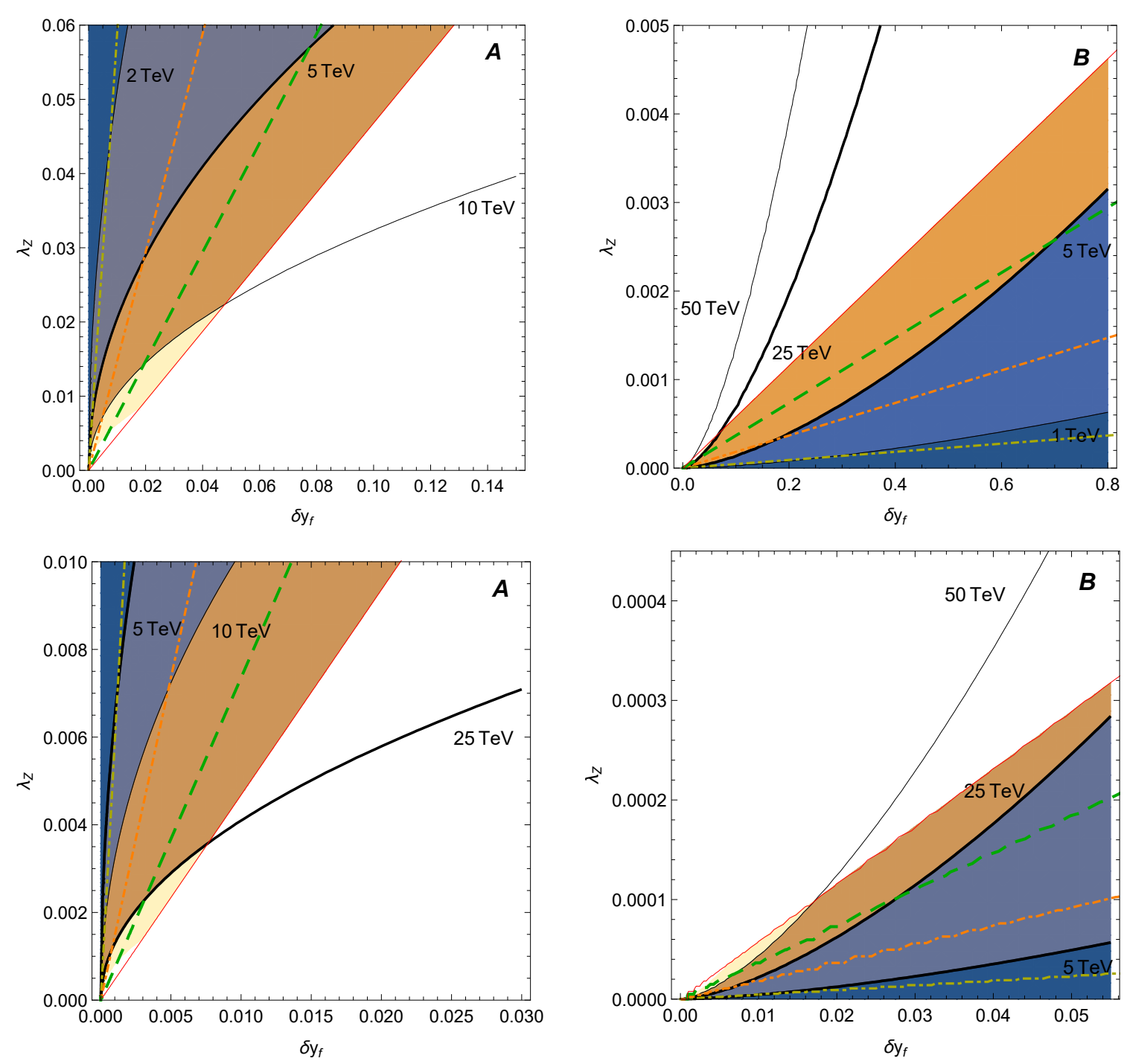

Figure 3. Estimated (maximal) NP mass $M_{\max }$ in dependence on the variation in the Yukawa couplings $\left(\delta y_{f}\right)$ and the triple-gauge coupling $\left(\lambda_{Z}\right)$ in Scenario $\mathbf{A}$ (left) and $\mathbf{B}$ (right). The colored lines denote NP couplings of $g_{*}=1,4,8,4 \pi$, respectively (from yellow to red), and the lower panel shows a zoom into the plots of the upper panel. See text for details.

in the lower panel of figure 2 , where we again set $N_{\mathrm{UV}} \rightarrow 1$. As discussed before, in this case there are actual experimental hints for a non-vanishing NP effect, corresponding to $C_{9} \sim-1$, which we depict by the red dashed line. Motivated by this anomaly, we now exemplify concretely a potential determination of the ballpark of a NP mass $M$. Assume that in fact a value of $C_{9}=-1.2 \pm 0.2$ is established in the future [41], while the trilinear Higgs self-coupling exhibits a $\delta \lambda=(60 \pm 10) \%$ correction. Given this information, we could conclude that roughly $M \sim 50 \mathrm{TeV}$, which is determined by the lower right gray box, which shall sketch the experimental error. On the contrary, a hypothetical value of $C_{9}=-4 \pm 0.2$, together with the constraint $\delta \lambda \lesssim 10 \%$ would lead to the vastly different estimation $M \lesssim 5 \mathrm{TeV}$, if the underlying framework is Scenario $\mathbf{A}$. 
Now, we consider a simultaneous measurement of the TGC parameter $\lambda_{Z}$ and $\delta y_{f}$ in Scenario A. Here, a measurement of small $\delta y_{f}$ together with large $\lambda_{Z}$ would lead to LHC detectability of new states. For example, $\delta y_{f}=5 \%$ with $\lambda_{Z}=0.06$, being considerably on the top-left of the $M_{\max } \sim 5 \mathrm{TeV}$ line in the first plot in figure 3, should result in an LHC discovery in the scenario considered. However, such large values of $\lambda_{Z}$ are in fact excluded to date. In consequence, considering that $\lambda_{Z}<0.03$ we can already conclude now that the discovery of a deviation in Yukawa couplings at the LHC would on the other hand strongly disfavor detectability of the corresponding new states at the same collider, since the limits on the TGC would require the effects to come from larger masses (under the given assumptions)!

However, a measurement of $\delta y_{f}=1 \%$ at the ILC, together with a value as small as $\lambda_{Z} \sim 0.01$, would lead to $M_{\max } \sim 10 \mathrm{TeV}$ and thus strongly indicate a discovery at the FCC in Scenario A, see the lower left plot of the figure which zooms into the region of small effects.

In Scenario B, visualized in the right plots of figure 3, the scaling of $M_{\max }$ with $\lambda_{Z}$ is inverted, due to the additional loop factor entering $c_{3 V}$. Here, smaller $\lambda_{Z}$ lead to smaller $M_{\max }$ and a measurement of $\lambda_{Z}=0.001$, in LHC reach, together with a largish $\delta y_{f}=40 \%$ would indicate NP producible at the LHC, while more modest effects of $\delta y_{f}=5 \%$ would at least signal a NP state within direct FCC reach if they come together with small $\lambda_{Z} \sim 10^{-4}$ (testable at ILC). We should however note that even such values of $\lambda_{Z}$ are disfavored in Scenario $\mathbf{B}$, as we will see below - unless an unusual flavor structure is at work.

Large effects in $\lambda_{Z}$ together with reasonable $\delta y_{f}$, such as $\lambda_{Z} \gtrsim 0.2 \%$ and $\delta y_{f} \lesssim 50 \%$ are not possible in the perturbative regime, while the inverted case of, e.g., $\lambda_{Z} \lesssim 2 \times 10^{-4}$ and $\delta y_{f} \gtrsim 50 \%$ is in conflict with the non-observation of new sub-TeV particles. In general, the method thus provides a powerful means to test the consistency of the SILH assumption.

Another interesting pair of (pseudo-)observables is $\delta \lambda$ and $\lambda_{Z}$, explored in figure 4 . In Scenario $\mathbf{A}$, given in the left panel of the figure, large corrections to the trilinear Higgs self coupling are viable, with little impact on $\lambda_{Z}$. Observing for example a $\delta \lambda=200 \%$ correction simultaneously with $\lambda_{Z}=4 \times 10^{-3}$ is consistent with the framework (with $N_{\mathrm{UV}}=$ 3 ) and corresponds to a very large NP mass beyond LHC reach of $M_{\max } \sim 20 \mathrm{TeV}$, which could however be found at the FCC. On the other hand, seeing $\delta \lambda=50 \%$ simultaneously with $\lambda_{Z}=0.03$ leads to $M_{\max }<4 \mathrm{TeV}$ (with $g_{*}>1$ ), which would suggest a new particle within LHC reach.

In Setup B, on the other hand, a potential ILC extraction of $\delta \lambda \sim 10 \%$ would be required in order to make a meaningful prediction (see right panel of the figure), which, depending on $\lambda_{Z}$, could lead either to LHC or FCC detectability - the former for $\lambda_{Z} \gtrsim 0.03$ and the latter for $\lambda_{Z}$ approaching $10^{-2}$. However, we recall from figure 2 that a measurable $\delta \lambda$ is in fact disfavored in Scenario $\mathbf{B}$, unless the flavor structure leading to $C_{9}$ would not follow the assumed scaling.

The remaining plane to be discussed is spanned by $C_{9}$ and $\lambda_{Z}$. Here we can focus on Scenario A, depicted in the left panel of figure 5 , since measurable effects in Scenario $\mathbf{B}$ in $\lambda_{Z}$, exceeding $10^{-4}$, are basically excluded from perturbativity (for $C_{9} \lesssim \mathcal{O}(1)$ ) under the given assumptions, as explored in the right panel of the same figure. A sizable $\lambda_{Z}$ would 

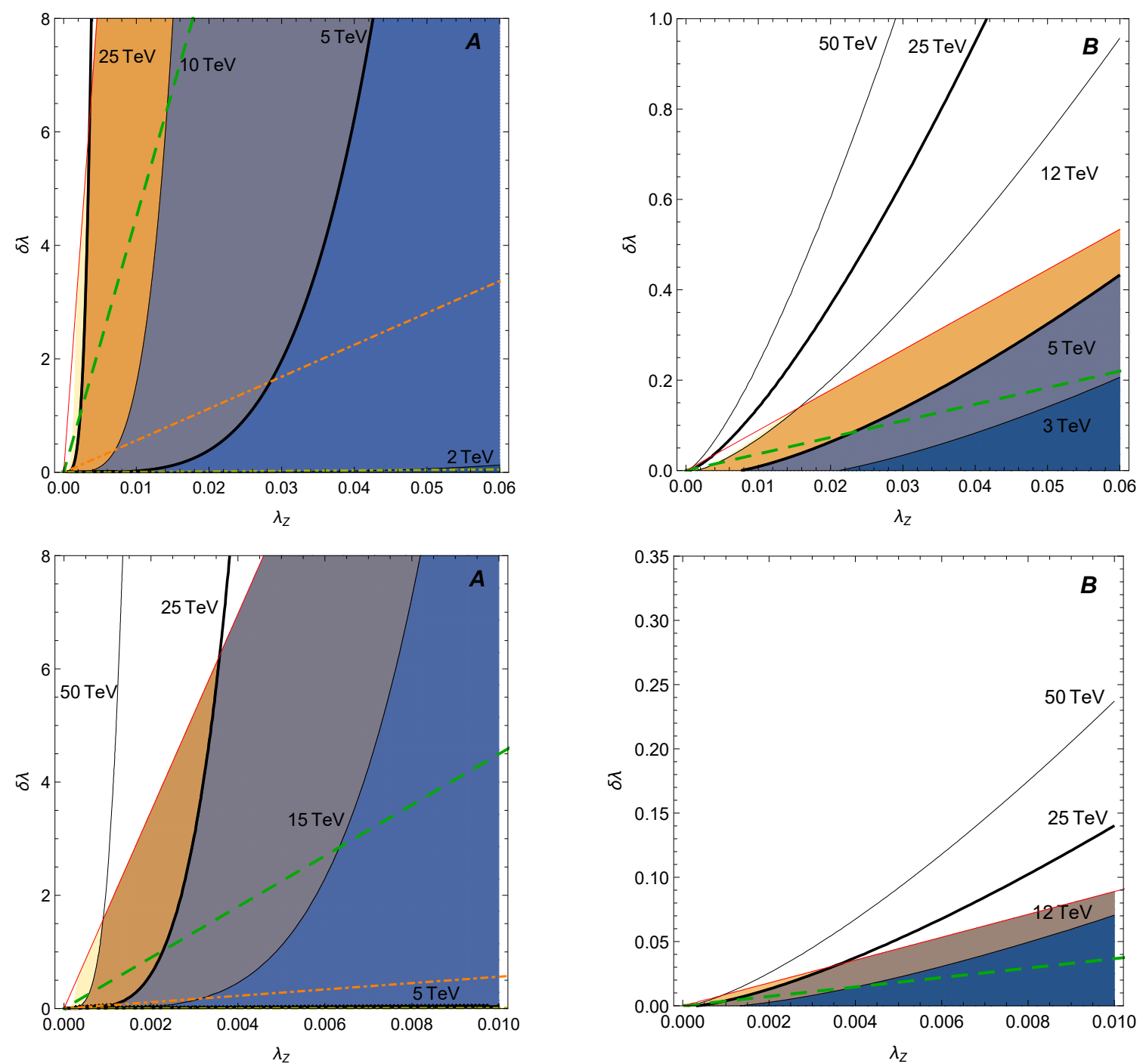

Figure 4. Estimated (maximal) NP mass $M_{\max }$ in dependence on the triple-gauge coupling $\left(\lambda_{Z}\right)$ and the variation in the triple-Higgs self coupling $(\delta \lambda)$ in Scenario $\mathbf{A}$ (left) and $\mathbf{B}$ (right). The colored lines denote NP couplings of $g_{*}=1,4,8,4 \pi$, respectively (from yellow to red), and the lower panel shows a zoom into the plots of the upper panel. See text for details.

in fact exclude this scenario. In the former setup, however, largely different NP masses can be accessed. While $\lambda_{Z}=0.02$ with $C_{9}=-1$ leads to the estimate $M_{\max } \sim 2 \mathrm{TeV}$, just at the boundary of the current direct reach, for example $\lambda_{Z}=0.005$ with $C_{9}=-3$ would lead to $M_{\max } \sim 15 \mathrm{TeV}$, beyond LHC but within FCC reach.

Before concluding we will finally briefly explore the procedure lined out below eq. (3.2) for a simple toy example, keeping in mind the caveats regarding $\mathcal{O}(1)$ factors discussed in detail. To this end, consider first a measurement of $\lambda_{Z} \approx 0.15 \%$ and $\delta \lambda \approx 10 \%{ }^{15}$ Employing eq. (3.2), we deduce $M \approx 18 \mathrm{TeV}$ for Scenario $\mathbf{B}$ and $M \approx 9 \mathrm{TeV}$ for Scenario A, with couplings $g_{*} \approx 10$ and $g_{*} \approx 2$, respectively. With this information, we derive

\footnotetext{
${ }^{15}$ Although this is a far-future scenario, it serves the illustrative purpose.
} 

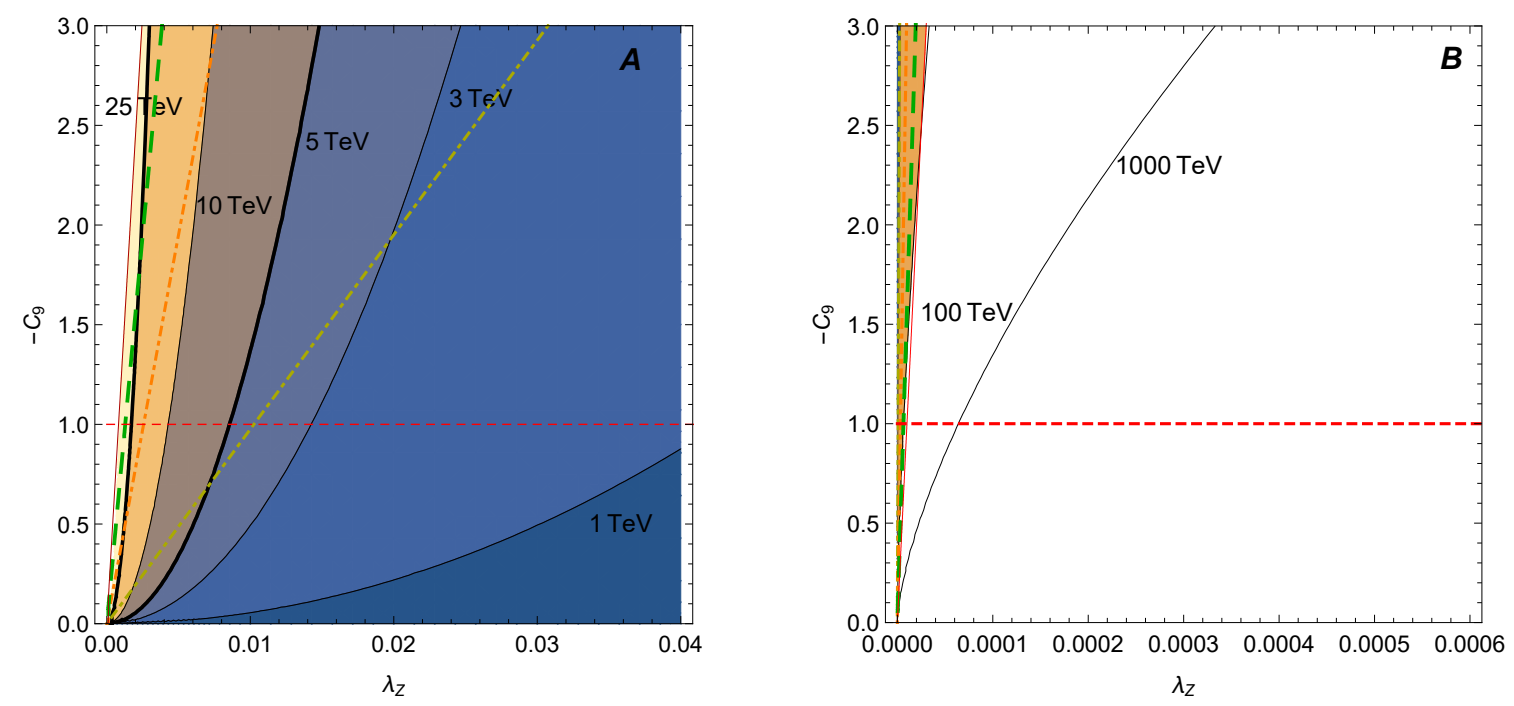

Figure 5. Estimated (maximal) NP mass $M_{\max }$ in dependence on the triple-gauge coupling $\left(\lambda_{Z}\right)$ and the coefficient of the four fermion operator $\mathcal{O}_{9}\left(C_{9}\right)$ in Scenario $\mathbf{A}$ (left) and $\mathbf{B}$ (right). The colored lines denote NP couplings of $g_{*}=1,4,8,4 \pi$, respectively (from yellow to red), and the horizontal dashed line depicts the currently preferred value of $C_{9} \sim-1$. See text for details.

via eqs. (3.1) and (3.2) $\delta y_{f} \approx 3 \%$ with $C_{9} \approx-18$ in Scenario $\mathbf{B}$, while in Scenario A we obtain $\delta y_{f} \approx 0.5 \%$ and $C_{9} \approx-2$. So while Scenario $\mathbf{B}$ seems disfavored, establishing a $C_{9} \lesssim-(1-2)$ would lead to a very consistent picture of effects in Scenario $\mathbf{A}$ with NP probably beyond direct LHC reach.

\section{Conclusions}

We have shown how simultaneous measurements of (pseudo-)observables allow to estimate whether the NP could be expected to materialize at current or future colliders. This is achieved by exploiting their different scaling with the NP coupling $g_{*}$, as derived after restoring the $\hbar$ dimensions of the operators, with the details of the procedure worked out in the body of the paper.

To summarize the results from the perspective of expected effects in the two orthogonal scenarios considered, in the ALH-like Scenario $\mathbf{A} \mathcal{O}(1)$ effects are possible in $\delta \lambda$, without other problematic contributions, while significant $\delta y_{f}$ of $\gtrsim 5 \%$ require a large $\lambda_{Z}$. In turn, sizable $C_{9}$ are possible without inducing large corrections to other pseudo-observables. Finally, a large $\lambda_{Z}$ at the per cent level induces also a non-negligible $\left|C_{9}\right| \gtrsim \mathcal{O}(1)$, unless the mass of the new physics is very low $\left(M_{\max } \lesssim(2-3) \mathrm{TeV}\right)$, about to be detected at the LHC.

On the other hand, the SILH-like Scenario $\mathbf{B}$ predicts basically tiny $\lambda_{Z}$ and $\delta \lambda$, below any detectable level, since otherwise $\left|C_{9}\right|$ becomes too large (requiring perturbativity). Even ignoring the flavor-structure related $C_{9}$, sizable $\lambda_{Z}$ and $\delta \lambda$ at a level detectable in the near future are disfavored, since they also induce large corrections in Yukawa couplings around or beyond the current experimental sensitivity. Finding however a sizable $\delta y_{f} \sim$ $30 \%$ would in turn lead to a $\lambda_{Z}$ probably observable in the long-term LHC run, if no direct 


\begin{tabular}{|c|c|c|c|c|}
\hline $\mathbf{A}$ & $\delta y_{f}$ & $\delta \lambda$ & $\lambda_{Z}$ & $c_{9}$ \\
\hline$\delta y_{f}$ & - & $25 \mathrm{TeV}$ & $25 \mathrm{TeV}$ & - \\
\hline$\delta \lambda$ & $25 \mathrm{TeV}$ & - & $70 \mathrm{TeV}$ & $150 \mathrm{TeV}$ \\
\hline$\lambda_{Z}$ & $25 \mathrm{TeV}$ & $70 \mathrm{TeV}$ & - & $70 \mathrm{TeV}$ \\
\hline$C_{9}$ & - & $150 \mathrm{TeV}$ & $70 \mathrm{TeV}$ & - \\
\hline
\end{tabular}

\begin{tabular}{|c|c|c|c|c|}
\hline $\mathbf{B}$ & $\delta y_{f}$ & $\delta \lambda$ & $\lambda_{Z}$ & $c_{9}$ \\
\hline$\delta y_{f}$ & - & $8 \mathrm{TeV}$ & $25 \mathrm{TeV}$ & - \\
\hline$\delta \lambda$ & $8 \mathrm{TeV}$ & - & $8 \mathrm{TeV}$ & N/A \\
\hline$\lambda_{Z}$ & $25 \mathrm{TeV}$ & $8 \mathrm{TeV}$ & - & $40 \mathrm{TeV}$ \\
\hline$C_{9}$ & - & N/A & $40 \mathrm{TeV}$ & - \\
\hline
\end{tabular}

Table 4. Estimate of ballpark for maximal mass $M$ detectable, for each pair of pseudo-observables, considering ILC projections, see text for details.

discovery at the LHC is made (i.e., assuming $M_{\max } \gtrsim 5 \mathrm{TeV}$ ) and to a $C_{9}$ vastly exceeding limits. The strong and correlated predictions in this scenario offer a powerful means to test it indirectly in the near future with simple observations, such as to understand the nature of NP. Finally, sizable $C_{9}$ are viable in Scenario $\mathbf{B}$, without any other observable prediction.

After all, this work provides on overview of which patterns of NP can be expected and how an indication for the NP mass can be obtained, given only indirect observation, which can help to find the UV completion of the SM and to develop strategies to detect the NP directly.

\section{Acknowledgments}

I am indebted to Roberto Contino, Adam Falkowski, Christophe Grojean, and Francesco Riva for valuable discussions on EFT extensions of the SM and to Adam Falkowski and Francesco Riva for useful remarks on the manuscript. Finally, I thank the CERN theory division for the wonderful time during my fellowship (2014-16), when most of this work was performed.

Open Access. This article is distributed under the terms of the Creative Commons Attribution License (CC-BY 4.0), which permits any use, distribution and reproduction in any medium, provided the original author(s) and source are credited.

\section{References}

[1] W. Buchmüller and D. Wyler, Effective Lagrangian analysis of new interactions and flavor conservation, Nucl. Phys. B 268 (1986) 621 [INSPIRE].

[2] K. Hagiwara, S. Ishihara, R. Szalapski and D. Zeppenfeld, Low-energy effects of new interactions in the electroweak boson sector, Phys. Rev. D 48 (1993) 2182 [INSPIRE].

[3] B. Grzadkowski, M. Iskrzynski, M. Misiak and J. Rosiek, Dimension-six terms in the Standard Model Lagrangian, JHEP 10 (2010) 085 [arXiv: 1008.4884] [INSPIRE].

[4] S. Weinberg, Dynamical approach to current algebra, Phys. Rev. Lett. 18 (1967) 188 [INSPIRE].

[5] S. Weinberg, Nonlinear realizations of chiral symmetry, Phys. Rev. 166 (1968) 1568 [INSPIRE]. 
[6] S.R. Coleman, J. Wess and B. Zumino, Structure of phenomenological Lagrangians. 1, Phys. Rev. 177 (1969) 2239 [INSPIRE].

[7] C.G. Callan Jr., S.R. Coleman, J. Wess and B. Zumino, Structure of phenomenological Lagrangians. 2, Phys. Rev. 177 (1969) 2247 [INSPIRE].

[8] R.F. Dashen, Chiral $\mathrm{SU}(3) \times \mathrm{SU}(3)$ as a symmetry of the strong interactions, Phys. Rev. 183 (1969) 1245 [INSPIRE].

[9] R.F. Dashen and M. WEinstein, Soft pions, chiral symmetry and phenomenological Lagrangians, Phys. Rev. 183 (1969) 1261 [inSPIRE].

[10] L.-F. Li and H. Pagels, Perturbation theory about a Goldstone symmetry, Phys. Rev. Lett. 26 (1971) 1204 [INSPIRE].

[11] K.G. Wilson, Renormalization group and critical phenomena. 1. Renormalization group and the Kadanoff scaling picture, Phys. Rev. B 4 (1971) 3174 [InSPIRE].

[12] K.G. Wilson, Renormalization group and critical phenomena. 2. Phase space cell analysis of critical behavior, Phys. Rev. B 4 (1971) 3184 [INSPIRE].

[13] K.G. Wilson and J.B. Kogut, The renormalization group and the $\epsilon$-expansion, Phys. Rept. 12 (1974) 75 [INSPIRE].

[14] T. Appelquist and J. Carazzone, Infrared singularities and massive fields, Phys. Rev. D 11 (1975) 2856 [INSPIRE].

[15] S. Weinberg, Phenomenological Lagrangians, Physica A 96 (1979) 327 [INSPIRE].

[16] S. Weinberg, Baryon and lepton nonconserving processes, Phys. Rev. Lett. 43 (1979) 1566 [INSPIRE].

[17] F. Wilczek and A. Zee, Operator analysis of nucleon decay, Phys. Rev. Lett. 43 (1979) 1571 [INSPIRE].

[18] S. Weinberg, Effective gauge theories, Phys. Lett. B 91 (1980) 51 [InSPIRE].

[19] H.A. Weldon and A. Zee, Operator analysis of new physics, Nucl. Phys. B 173 (1980) 269 [INSPIRE].

[20] J. Polchinski, Effective field theory and the Fermi surface, in Proceedings, Theoretical Advanced Study Institute (TASI 92): from black holes and strings to particles, Boulder, CO, U.S.A., 1-26 June 1992, pg. 235 [NSF-ITP-92-132] [hep-th/9210046] [INSPIRE].

[21] H. Georgi, Effective field theory, Ann. Rev. Nucl. Part. Sci. 43 (1993) 209 [inSPIRE].

[22] A. Pich, Effective field theory: course, in Probing the Standard Model of particle interactions. Proceedings, Summer School in Theoretical Physics, NATO Advanced Study Institute, $68^{\text {th }}$ session, Les Houches, France, 28 July-5 September 1997, pg. 949 [hep-ph/9806303] [INSPIRE].

[23] A.J. Buras, Weak Hamiltonian, CP-violation and rare decays, in Probing the Standard Model of particle interactions. Proceedings, Summer School in Theoretical Physics, NATO Advanced Study Institute, $68^{\text {th }}$ session, Les Houches, France, 28 July-5 September 1997, pg. 281 [hep-ph/9806471] [INSPIRE].

[24] M. Neubert, Effective field theory and heavy quark physics, in Physics in $D \geq 4$. Proceedings, Theoretical Advanced Study Institute in elementary particle physics, TASI 2004, Boulder, CO, U.S.A., 6 June-2 July 2004, World Scientific, Singapore (2005), pg. 149 [hep-ph/0512222] [INSPIRE]. 
[25] C.P. Burgess, Introduction to effective field theory, Ann. Rev. Nucl. Part. Sci. 57 (2007) 329 [hep-th/0701053] [INSPIRE].

[26] A. Falkowski, Effective field theory approach to LHC Higgs data, Pramana 87 (2016) 39 [arXiv: 1505.00046] [INSPIRE].

[27] LHC Higgs Cross Section Working Group collaboration, Handbook of LHC Higgs cross sections: 4. Deciphering the nature of the Higgs sector, arXiv:1610.07922 [INSPIRE].

[28] A.V. Manohar, Introduction to effective field theories, in Les Houches summer school: EFT in particle physics and cosmology, Les Houches, France, 3-28 July 2017 [arXiv:1804.05863] [INSPIRE].

[29] G.F. Giudice and M. McCullough, A clockwork theory, JHEP 02 (2017) 036 [arXiv: 1610.07962] [INSPIRE].

[30] G.F. Giudice, C. Grojean, A. Pomarol and R. Rattazzi, The strongly-interacting light Higgs, JHEP 06 (2007) 045 [hep-ph/0703164] [INSPIRE].

[31] M.A. Luty, Naive dimensional analysis and supersymmetry, Phys. Rev. D 57 (1998) 1531 [hep-ph/9706235] [INSPIRE].

[32] A.G. Cohen, D.B. Kaplan and A.E. Nelson, Counting 4 $\pi$ 's in strongly coupled supersymmetry, Phys. Lett. B 412 (1997) 301 [hep-ph/9706275] [INSPIRE].

[33] R. Contino, A. Falkowski, F. Goertz, C. Grojean and F. Riva, On the validity of the effective field theory approach to SM precision tests, JHEP 07 (2016) 144 [arXiv:1604.06444] [INSPIRE].

[34] K. Agashe, R. Contino and A. Pomarol, The minimal composite Higgs model, Nucl. Phys. B 719 (2005) 165 [hep-ph/0412089] [INSPIRE].

[35] D. Liu, A. Pomarol, R. Rattazzi and F. Riva, Patterns of strong coupling for LHC searches, JHEP 11 (2016) 141 [arXiv: 1603.03064] [INSPIRE].

[36] J. de Blas, M. Chala, M. Pérez-Victoria and J. Santiago, Observable effects of general new scalar particles, JHEP 04 (2015) 078 [arXiv: 1412.8480] [INSPIRE].

[37] B. Henning, X. Lu and H. Murayama, How to use the Standard Model effective field theory, JHEP 01 (2016) 023 [arXiv:1412.1837] [INSPIRE].

[38] G. D'Ambrosio, G.F. Giudice, G. Isidori and A. Strumia, Minimal flavor violation: an effective field theory approach, Nucl. Phys. B 645 (2002) 155 [hep-ph/0207036] [INSPIRE].

[39] S. Descotes-Genon, J. Matias and J. Virto, Understanding the $B \rightarrow K^{*} \mu^{+} \mu^{-}$anomaly, Phys. Rev. D 88 (2013) 074002 [arXiv:1307.5683] [INSPIRE].

[40] R. Gauld, F. Goertz and U. Haisch, On minimal $Z^{\prime}$ explanations of the $B \rightarrow K^{*} \mu^{+} \mu^{-}$ anomaly, Phys. Rev. D 89 (2014) 015005 [arXiv: 1308.1959] [INSPIRE].

[41] W. Altmannshofer, C. Niehoff, P. Stangl and D.M. Straub, Status of the $B \rightarrow K^{*} \mu^{+} \mu^{-}$ anomaly after Moriond 2017, Eur. Phys. J. C 77 (2017) 377 [arXiv:1703.09189] [INSPIRE].

[42] G. D'Amico et al., Flavour anomalies after the $R_{K^{*}}$ measurement, JHEP 09 (2017) 010 [arXiv: 1704.05438] [INSPIRE].

[43] E. Megias, G. Panico, O. Pujolàs and M. Quirós, A natural origin for the LHCb anomalies, JHEP 09 (2016) 118 [arXiv: 1608.02362] [INSPIRE]. 
[44] M.E. Peskin, Estimation of LHC and ILC capabilities for precision Higgs boson coupling measurements, in Proceedings, 2013 Community Summer Study on the Future of U.S. Particle Physics: Snowmass on the Mississippi (CSS2013), Minneapolis, MN, U.S.A., 29 July-6 August 2013 [arXiv: 1312.4974] [INSPIRE].

[45] F. Goertz, A. Papaefstathiou, L.L. Yang and J. Zurita, Higgs boson self-coupling measurements using ratios of cross sections, JHEP 06 (2013) 016 [arXiv:1301.3492] [INSPIRE].

[46] F. Goertz, A. Papaefstathiou, L.L. Yang and J. Zurita, Higgs boson pair production in the $D=6$ extension of the SM, JHEP 04 (2015) 167 [arXiv:1410.3471] [INSPIRE].

[47] A. Azatov, R. Contino, G. Panico and M. Son, Effective field theory analysis of double Higgs boson production via gluon fusion, Phys. Rev. D 92 (2015) 035001 [arXiv:1502.00539] [INSPIRE].

[48] L. Bian, J. Shu and Y. Zhang, Prospects for triple gauge coupling measurements at future lepton colliders and the $14 \mathrm{TeV}$ LHC, JHEP 09 (2015) 206 [arXiv: 1507.02238] [INSPIRE].

[49] A. Azatov, J. Elias-Miro, Y. Reyimuaji and E. Venturini, Novel measurements of anomalous triple gauge couplings for the LHC, JHEP 10 (2017) 027 [arXiv: 1707.08060] [INSPIRE].

[50] G. Panico, F. Riva and A. Wulzer, Diboson interference resurrection, Phys. Lett. B 776 (2018) 473 [arXiv: 1708.07823] [INSPIRE].

[51] D.M. Asner et al., ILC Higgs white paper, in Proceedings, 2013 Community Summer Study on the Future of U.S. Particle Physics: Snowmass on the Mississippi (CSS2013), Minneapolis, MN, U.S.A., 29 July-6 August 2013 [arXiv:1310.0763] [INSPIRE].

[52] S.-F. Ge, H.-J. He and R.-Q. Xiao, Probing new physics scales from Higgs and electroweak observables at $e^{+} e^{-}$Higgs factory, JHEP 10 (2016) 007 [arXiv: 1603.03385] [INSPIRE].

[53] S.-F. Ge, H.-J. He and R.-Q. Xiao, Testing Higgs coupling precision and new physics scales at lepton colliders, Int. J. Mod. Phys. A 31 (2016) 1644004 [arXiv:1612. 02718] [InSPIRE].

[54] Y. Gershtein et al., Working group report: new particles, forces and dimensions, in Proceedings, 2013 Community Summer Study on the Future of U.S. Particle Physics:

Snowmass on the Mississippi (CSS2013), Minneapolis, MN, U.S.A., 29 July-6 August 2013 [arXiv: 1311.0299] [INSPIRE].

[55] L. Di Luzio, R. Gröber and M. Spannowsky, Maxi-sizing the trilinear Higgs self-coupling: how large could it be?, Eur. Phys. J. C 77 (2017) 788 [arXiv:1704.02311] [InSPIRE]. 